\title{
MID-TERM RESULTS OF AUTOINJECTION
}

\section{THERAPY FOR ERECTILE DYSFUNCTION}

\author{
CHRISTIAN G. STIEF, M.D. \\ HELMUTH GALL, M.D. \\ WOLFGANG SCHERB, M.D. \\ WOLFGANG BÄHREN, M.D.
}

From the Departments of Urology, Dermatology, Neurology, and

Radiology, Bundeswehrkrankenhaus, Academic Hospital of the

University of Ulm, Ulm, West Germany

\begin{abstract}
Of over 300 patients with erectile dysfunction, 186 were selected for intracavernosal autoinjection therapy with a standardized papaverine-phentolamine mixture. A total of 156 patients performed 4,813 protocol autoinjections with a minimum of 10 and a maximum of 230 per patient. The dose that induced a full erection at the hospital could be reduced under home conditions by a mean of 35 per cent. Systemic side effects were not observed. The most inconvenient local side effects were prolonged erections in 24 patients in diagnostic use and in 3 patients in therapeutic use. These were treated easily without further consequences.
\end{abstract}

In 1974, Klinge and Sjöstrand, ${ }^{1}$ from Scandinavia, examined the effects of various neurotransmitters and vasoactive drugs on the musculus retractor penis and the arteria penis of the bull. Virag'2 reported in 1982 on the periodic intracavernosal application of papaverine in man, that restored erectile potency in $4 / 14$ patients. One year later, Brindley ${ }^{3}$ described the demand-oriented intracavernosal injection of the alpha-receptor-blockade, phenoxyben-

TABLE I. Exclusion criteria for diagnostic and therapeutic use of intracavernous injection of vasoactive drugs
Age over 65 years
Cardiac dysrhythmias
AOD Stages III and IV
Addiction
Severe liver or kidney disease
Sexual deviation
Duration of erectile dysfunction less than one year
Unreliable and noncooperative patient

zamine. In 1984, Zorgniotti and Lefleur ${ }^{4}$ introduced autoinjection therapy with a vasoactive drug combination for erectile dysfunction.

Meanwhile, intracavernosal injection of vasoactive drugs has found widespread use in diagnosis and therapy of erectile dysfunction..$^{5-8}$ Long-term experience with this therapy is lacking, but side effects of other application forms, such as severe liver changes after i.v. papaverine, call for close observation of possible local or systemic side effects.

\section{Material and Methods}

Between February, 1985, and January, 1987, over 300 patients with erectile dysfunction lasting over one year underwent multidisciplinary examinations at our hospital to elucidate the origin of their erectile failure. Since vasoactive substances were to be used for diagnosis, patients with arterio-occlusion (AOD) (Stages III and IV), cardiac dysrhythmias, severe liver or kidney diseases, or patients older than sixty-five years were excluded because adverse reactions were anticipated (Table I). 
TABLE II. Acceptance of autoinjection therapy

\begin{tabular}{lcc}
\hline & \multicolumn{2}{c}{ Per Cent of- } \\
\cline { 2 - 3 } Acceptance & Patients & Partners \\
\hline Very good & 42.7 & 41.2 \\
Good & 26.0 & 29.8 \\
Satisfied & 23.8 & 25.2 \\
Less satisfied & 6.0 & 3.8 \\
Dissatisfied & 1.5 & $\ldots$ \\
\hline
\end{tabular}

The following tests were performed: anamnesis with the aid of a questionnaire, physical examination, blood tests (fats, testosterone, and prolactin), sexual anamnesis and psychometry (MMPI), bulbocavernosus reflex (BCR), somatosensory evoked potential (SSEP) of the dorsal nerve of the penis, ultrasonography of the large vessels, Doppler ultrasound study of the penile arteries, and penile brachial pressure index (PBI). Selective penile angiography was performed on 59 patients and dynamic cavernosography on 54 .

Subsequently, intracavernosal standardized pharmacotesting was done. After informed consent was obtained, each patient received an injection of papaverine hydrochloride $7.5 \mathrm{mg}$ and phentolamine mesylate $0.25 \mathrm{mg}$ into the proximal right corpus cavernosum by means of a 26-G needle. This dose was reduced or doubled the following day(s), depending on the rigidity obtained, until a full erection was induced (max. $45 \mathrm{mg}$ papaverine $+1.5 \mathrm{mg}$ phentolamine). This standardized pharmacotesting proved to be helpful in the differential diagnosis of vascular penile disturbances. ${ }^{\ominus}$

Of these patients, 186 were carefully selected for intracavernosal autoinjection therapy at home. Patients with sexual deviation or addiction were excluded. The levels of reliability and cooperation of the patients were very important. The dose was adapted to induce an erection of thirty to sixty minutes under hospital conditions. The patients were advised to reduce this dose, if the duration of full erection exceeded ninety minutes at home. The patients were reviewed after 10 and then all 25 autoinjections. Blood chemistry and ultrasound study of the penis were performed after all 50 injections. In case of a prolonged erection (after 4 hours), they were advised to return to the hospital immediately.

\section{Results}

In a follow-up period of from three to twenty-four months, 156 patients performed
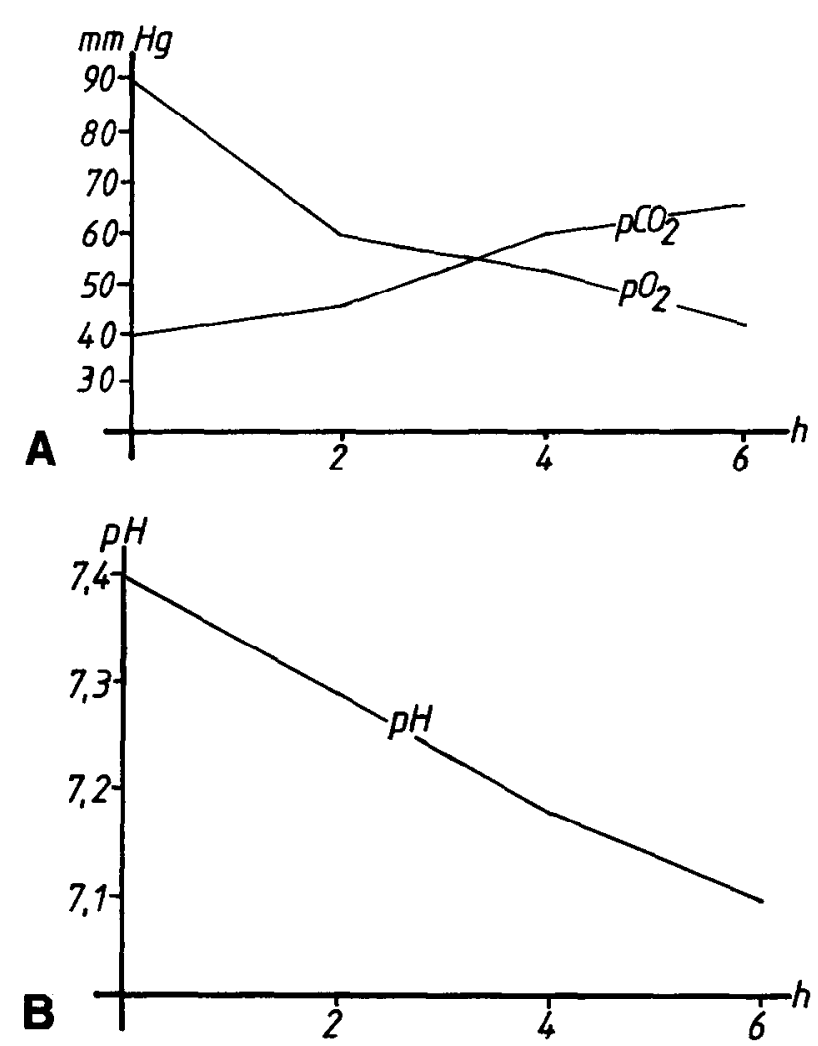

Figure 1. (A and B) Intracavernosal blood gas analysis after two hours $(n=4)$, four hours $(n=4)$, and six hours $(n=4)$ of full erection induced by vasoactive drugs.

4,813 protocol autoinjections with a minimum of 10 autoinjections per patient. A total of 52 of 156 patients performed more than 35 injections, 21 of 156 more than 100 , and 7 of 156 more than 200 injections at home. Fourteen patients reported a significant improvement in spontaneous erections with full erections up to fourteen days after the injection. Six patients regained spontaneous erections within the first 10 injections (4 psychogenic, 2 mild arterial). Ten patients abandoned this therapy (Table II). The patients reduced the individually adapted dose in hospital under home conditions by a mean of 35 per cent $(1.27 \mathrm{~mL}$ to 0.89 $\mathrm{mL})$. No patient reported systemic side effects. In 40 consecutive patients, continuous monitoring of pulse and blood pressure showed no influence on these cardiovascular parameters after the intracorporeal injection. No significant changes in blood chemistry were observed. In 3 patients with 35 to 60 autoinjections, a localized ( $<5 \mathrm{~mm}$ ) thickening of the tunica albuginea occurred. After eight weeks of noninjection, the thickening resolved in 3 of 3 patients. 
No cavernous fibrosis was detectable by palpation or ultrasound examinations.

In diagnostic use, 26 prolonged erections (pharmacologically induced erection) occurred in 24 patients. Because they involve the risk of ischemic damage to the cavernous tissue with resulting irreversible erectile impotence, ${ }^{10}$ prolonged erections should be stopped after a maximum of six hours (Fig. 1). The first 4 patients were treated by puncture and aspiration, the others by administration of $2.5 \mathrm{mg}$ or $2 \mathrm{mg}$ of metaraminol intracavernosally.

During autoinjection therapy, 3 prolonged erections occurred within the first 1,000 applications. In each case, the patient had injected another dose after he had thought the first to be unsuccessful. Intracavernosal injections of 2.5 $\mathrm{mg}$ metaraminol into the right and $1 \mathrm{mg}$ into the left corpus cavernosa led to complete flaccidity. Ever since we warned our patients not to administer more than 1 injection per day, no prolonged erections during home treatments were reported. In none of the patients were fibrqtic changes of the corporeal tissue, cavernitis, penile deviation, or anaphylactoid reaction observed. Intradermal hematomas (2$5 \mathrm{~mm}$ ) occurred with 2 to 3 per cent of the injections and were resorbed without consequences.

\section{Comment}

The most inconvenient short-term side effect of therapy with intracavernosal vasoactive drugs is prolonged erections. Various authors report a frequency of about 5 to 10 per cent in diagnostic use. ${ }^{5-8,10}$ Therefore, the patient should be in close contact with the urologist, especially at the beginning of therapy, and prolonged erections must be interrupted after a maximum of six hours.

Systemic side effects on the cardiovascular system were absent objectively and subjectively in our patients. This is probably due to the small doses we administer (mean $19.05 \mathrm{mg}$ papaverine $+0.64 \mathrm{~g}$ phentolamine). Other authors who reported occasional systemic side effects such as dizziness, inject up to $160 \mathrm{mg}$ papaverine. ${ }^{5,11}$
No cavernous fibrosis due to autoinjection therapy, as reported by other authors, ${ }^{12,13}$ was seen in our patients, even in those with more than 200 injections. Our drug mixture has a $\mathrm{pH}$ 4.5 whereas papaverine hydrochloride has a $\mathrm{pH}$ 2.7. Further follow-up is necessary to reveal the incidence of intracavernosal fibrosis in our patients.

In our opinion, the requirements for diagnosis and therapy of erectile dysfunction with vasoactive drugs are (1) the preselection and cooperation of the patient, (2) a physiologically adapted mixture, and (3) a urologist experienced in treating side effects.

Department of Urology, U 518 UCSF, School of Medicine San Francisco, California 94143

(DR. STIEF)

\section{References}

1. Klinge $\mathbf{E}$ and Sjöstrand NO: Contraction and relaxation of the retractor penis muscle and the penile artery of the bull, Acta Physiol Scand (Suppl) 420 (1974).

2. Virag R: Intracavernous injection of papaverine for erectile failure, Lancet 2: 938 (1982).

3. Brindley GS: Cavernosal alpha-blockade, $\mathrm{Br} \mathrm{J}$ Psychiatry 143: 332 (1983).

4. Zorgniotti AW, and Lefleur RS: Autoinjection of the corpus cavernosum with a vasoactive drug combination for vasculogenic impotence, J Urol 133: 39 (1985).

5. Sidi AA, Cameron JS, Duffy LM, and Lange PH: Intracavernous drug-induced erections in the management of male erectile dysfunction, J Urol 135: 704 (1986).

6. Dhabuwala CB, Jaikisken P, and Pierce JM: The effects of intracavernosal papaverine and phentolamine on penile erections (abstr. 592), J Urol, part 2, 133: 261 (1985).

7. Robinette MA, and Moffat MJ: Intracorporeal injection of papaverine and phentolamine in the management of impotence, Br J Urol 58: 692 (1986).

8. Stief CG, et al: Schwellkörper-Autoinjektions-Therapie (SKAT), Urologe [A] 25: 63 (1986).

9. Stief CG, et al: The meaningfulness of vasoactive drugs in the diagnosis of erectile dysfunction, presented at Second World Meeting on Impotence, Prague, 1986.

10. Lue TF, and Tanagho EA: Physiology of erection and pharmacological management of impotence, J Urol 137: 829 (1987).

11. Brindley GS: Pilot experiments on the actions of drugs injected into the human corpus cavernosum penis, $\mathrm{Br}$ J Pharmacol 87: 495 (1986).

12. Larsen EH, Gasser TC, and Bruskewitz RC: Fibrosis of corpus cavernosum after intracavernous injection of phentolamine/papaverine, J Urol 137: 292 (1987).

13. Zorgniotti AW: Corpus cavernosum blockade for impotence: practical aspects and results in 250 cases (abstr. 808), J Urol, part 2, 135: 306A (1986). 